\title{
Distributed Learning and Multiaccess of On-Off Channels
}

\author{
Shiyao Chen and Lang Tong
}

\begin{abstract}
The problem of distributed access of a set of $N$ onoff channels by $K \leq N$ users is considered. The channels are slotted and modeled as independent but not necessarily identical alternating renewal processes. Each user decides to either observe or transmit at the beginning of every slot. A transmission is successful only if the channel is at the on state and there is only one user transmitting. When a user observes, it identifies whether a transmission would have been successful had it decided to transmit. A distributed learning and access policy referred to as alternating sensing and access (ASA) is proposed. It is shown that ASA has finite expected regret when compared with the optimal centralized scheme with fixed channel allocation.

Index terms-Multiaccess, Distributed learning, Cognitive radio networks.
\end{abstract}

\section{INTRODUCTION}

$\mathbf{T}$ $\mathrm{HE}$ problem considered in this paper, in its more general form, is related to distributed allocation of $N$ independent and randomly available resources among $K$ agents. By distributed allocation we mean that there is no central controller assigning resources to agents, and each agent acts on its own without communicating with others. We are interested in whether there is a distributed access policy that, through taking actions and learning from outcomes of the actions, achieves the utilization of resources comparable to that of the optimal centralized allocation.

We study the above in the context of multiaccess of $N$ random on-off channels by $K$ independent users. We are interested in whether any distributed learning and access policy is necessarily penalized by the lack of coordination. The performance measure of interest is throughput-the fraction of time that transmissions are successful. For a $K$ user multiaccess system, the throughput is defined by a vector $\mathbf{r}=\left(r_{1}, \cdots, r_{K}\right)$ where $r_{i}$ is the throughput of user $i$. If $\mathbf{r}$ can be achieved by a central controller who assigns a channel to each user, we would like to achieve the same by letting users act independently on their own.

We should point out at the outset that when there are more users than the number of channels, i.e., $K>N$, the throughput region achievable by the optimal distributed access is, in general, strictly smaller than that by the optimal centralized scheme. This can be seen from the case when $N=1$, which corresponds to the classical random access problem. The centralized scheme achieves the maximum sum-rate of 1

Shiyao Chen and Lang Tong are with the School of Electrical and Computer Engineering, Cornell University, Ithaca, NY 14853. Email: $\{$ sc933, lt35\}ecornell. edu.

The work is supported in part by the National Science Foundation under award CCF 1018115 and the Army Research Office under award W911NF1010419. packet per slot. The celebrated slotted ALOHA protocol can be used to achieve, asymptotically as $K \rightarrow \infty$, the sum rate of $e^{-1}$. Although the optimal policy of distributed random access for this case is unknown, it is well known [1] that distributed random access cannot achieve the throughput of the optimal centralized channel allocation scheme.

Thus we restrict ourselves to the case when $N \geq K$. Here it is no longer obvious that distributed multiaccess performs strictly worse than the centralized counterpart. Because a user is not restricted to transmitting on one particular channel, it can search for opportunities elsewhere to avoid colliding with others. Intuitively, as $N$ increases, conflicts among users diminish, and users may be able to orthogonalize themselves to avoid collision. Even when $K=N$, a user can learn where other users are transmitting and act accordingly to avoid collision.

Beside collisions among competing users, we also consider a specific nontrivial channel imperfection. In particular, we assume that the channels are independent on/off random processes. Such a random channel model arises naturally from channel fading in wireless systems. In the context of multiaccess of cognitive radios [2], this model includes the situation when a channel is unavailable when it is occupied by another user of higher priority. In both cases, it is difficult for a user to identify whether the transmission failure is caused by collision with another user or by channel fading. Learning in such an uncertain environment cannot be perfect. It is therefore not obvious that mistakes in learning only cause negligible performance loss.

Like many online learning problems in uncertain environments, to achieve the best performance requires careful tradeoffs between exploration and exploitation. The results presented in this paper is an instance of such tradeoff that balances sensing and transmission.

\section{A. Summary of Results}

The detailed system model and assumptions are given in Section III Here we outline the context of the problem and summarize our main results. We consider $N$ independent but not necessarily identical on-off slotted channels. Our results apply to more general settings, but at the moment, it is sufficient to think these channels as independent Bernoulli channels with probability $\eta_{i}$ that the $i$ th channel is at the on state. Let $\boldsymbol{\eta}=\left(\eta_{1}, \ldots, \eta_{N}\right)$.

For a $K$ user multiaccess system, it is obvious that any point in

$$
\overline{\mathscr{R}}=\left\{\left(r_{1}, \ldots, r_{K}\right) \mid r_{(i)} \leq \eta_{(i)}, 1 \leq i \leq K\right\},
$$


can be achieved by a central controller with fixed channel allocation, where $r_{(i)}$ and $\eta_{(i)}$ are the ordered list of $r_{i}$ and $\eta_{i}$, respectively. Indeed $\overline{\mathscr{R}}$ is the largest achievable region by a central controller under fixed channel allocation without time sharing arrangement and without using channel state realizations.

The main result of this paper is to show that, under the model specified in Section $\amalg$ every point in $\overline{\mathscr{R}}$ is achievable by a decentralized access policy. This result is established by constructing a distributed learning and access policy executed independently by all users. The policy alternates between sensing and access periods, hence referred to as the alternating sensing and access (ASA) policy.

The throughput result above is a direct consequence of a more refined analysis based on the notion of regret between the total number of successful transmissions up to slot $n$ of the optimal centralized scheme $\bar{S}_{i}(n)$ and that of the distributed scheme $S_{i}(n)$ proposed here. We show in Theorem 1 that, if $\mathbf{r} \in \overline{\mathscr{R}}$, the expected regret of ASA approaches to a constant, i.e.,

$$
\mathbb{E}\left(\bar{S}_{i}(n)-S_{i}(n)\right) \sim O(1) .
$$

We should point out that when channels are homogeneous, i.e., $\eta_{i}=\eta$ for all $i, \overline{\mathscr{R}}$ is the largest throughput region achievable by a centralized controller, and ASA matches with the optimal centralized allocation. For heterogeneous channels, while $\overline{\mathscr{R}}$ is the largest throughput region by a centralized controller under fixed channel allocation, it is not necessarily convex. Therefore, $\overline{\mathscr{R}}$ may be enlarged by a central controller through time sharing.

In comparing with centralized access policies, we exclude the possibilities of time sharing arrangement, which is a loss of generality. Such a loss, however, is inevitable since the optimal distributed access policy cannot in general achieve the convexified throughput region 1 .

\section{B. Related Work}

The problem of orthogonalizing multiple coexisting users in a distributed manner through learning and individual actions has been studied as a decentralized learning of multi-armed bandit (MAB) processes involving multiple players in [3]. Essentially the same problem has also be studied for the multiaccess problem in multiuser cognitive radio systems [4], [5]. Further development of the prioritized and fair access cases is provided in [6], [7].

There are similarities and significant differences between these "MAB approaches" and that considered in this paper. The MAB formulation involves independent random processes, often assumed independent and identically distributed (iid) in time but may also be Markovian. Each process is associated with an unknown deterministic parameter. Lai and Robbins considered the single user (non-Bayesian) MAB

\footnotetext{
${ }^{1}$ This can be seen by considering a two channel case when $\eta_{1}=1$ and $\eta_{2}=\epsilon$. As $\epsilon \rightarrow 0$, a centralized access can accommodate a rate vector of $(0.5,0.5)$ by time sharing on a single channel, which cannot be achieved by any decentralized access policy.
}

problem, aiming to maximize the accumulated reward using knowledge learned from the outcome of past plays [8]. The problem falls in the category of "learning through doing".

The centralized multiuser version of the MAB problem was considered in [9] as a single user MAB problem but with the possibility of simultaneously playing multiple arms. The decentralized MAB problem was addressed explicitly in [3] and in the context of cognitive radio systems in [4], [5]. Typically, learning in the MAB problem refers to learning which arms are more favorable to play. The regret of the order optimal distributed learning with respect to the oracle player often increases with the number of plays as $O(\log n)$, unlike that in Eq. (2).

The problem considered in this paper does not belong to the category of MAB problem although it shares some common characteristics with the MAB formulation. We highlight here three main differences. First, unlike the MAB problems, the parameters of the underlying random processes are known. Thus there is little ambiguity on which channels are favorable for transmissions. Learning in this context deals with searching for appropriate channels to transmit, not knowing (for certain) the presence of other users.

Second, while the objective of MAB involves maximizing reward, we are interested in whether a set of rewards can be achieved through learning and transmission; each user does not try to maximize its throughput.

Third, the uncertainty associated with the presence of other users is a key distinction between the problem treated here and the MAB formulation. For the multiuser MAB problem, the presence of other players are certain whenever two players play the same arm. In our case, a failed transmission may be caused either by collision or by the fact that the channel is off.

A related problem is learning parameters of multiple independent processes when a user can choose where and when to observe a particular process [10]-[12]. Without actively engaging with other users, such formulations are more akin to the classical parameter estimation problems, not one of "learning through action" studied in our and the MAB formulations.

\section{System Model AND ASSUMPTIONS}

The multiaccess system considered includes $N$ channels, $K$ distributed users, and a basestation. We specify their roles in their interactions and assumptions made in this paper.

\section{A. Channel Model}

The $N$ channels are slotted and statistically independent. We consider a slot atomic, which means that it cannot be divided further so that multiple actions can be taken within one slot. The channel state of each channel in a slot is either "on" or "off" with "on" indicating that the channel can be used for transmission and "off" otherwise. The state of each channel is therefore a discrete-time binary process for which we model it as a renewal sequence alternating between consecutive "on" and "off" periods. 
The distribution of the on(off) period of channel $i$ is $F_{i}^{\text {on }}\left(F_{i}^{\text {off }}\right)$. We assume that $\mathbb{E} e^{\theta U}$ is well defined for some $t>0$, where $U$ is distributed as $F_{i}^{\text {on }}\left(F_{i}^{\text {off }}\right)$. Denote the mean of $F_{i}^{\text {on }}\left(F_{i}^{\text {off }}\right)$ by $\mu_{i}^{\text {on }}\left(\mu_{i}^{\text {off }}\right)$, and the long term fraction of on periods of channel $i$ by $\eta_{i}=\mu_{i}^{\text {on }} /\left(\mu_{i}^{\text {on }}+\mu_{i}^{\text {off }}\right)$.

\section{B. User Action and Feedback}

Users act independently and persistently, each aimed at achieving some fixed throughput target. They do not have a synchronized starting slot; they may enter the system at different times.

A user makes the decision either to access a channel or to sense a particular channel at the beginning of slots based on the outcomes of its own past actions. If the user decides to take the action of accessing channel $i$ in a slot, it transmits a packet to the basestation over channel $i$. If the action of observing channel $i$ is taken, it monitors channel $i$ in the slot.

When a user transmits over a particular channel to the basestation, it receives a binary feedback at the end of the slot from the basestation indicating whether the transmission is successful. When the transmission is successful, we call the channel over which the transmission occurred available, which means that the channel is at the on state and no other user transmits. The user receives a feedback symbol "a". The transmission fails if the channel is at the off state or when multiple transmissions occur at the same time. In this case, we call the channel unavailable, and the user receives a feedback symbol " $\bar{a}$ ". We note that the binary feedback does not specify which type of failure occurred to the transmission.

If a user decides to sense a particular channel in a slot, it observes the channel and decides whether the channel is available, i.e., whether a transmission would have been successful had the user decided to transmit. The outcome of the sensing action is again binary with " $a$ " indicating that the channel is available and " $\bar{a}$ " the opposite.

Note that the information obtained by a user in a slot through observation is identical to that through the feedback of a transmission. The difference is that there is a potential reward or damage caused by transmission.

\section{A Distributed LeARning And ACCess Policy}

In this section, we present a learning and access policy referred to as alternating sensing and access (ASA) policy. We show later in Section IV that ASA achieves the same throughput region as the optimal centralized scheme with fixed channel allocation.

The process of distributed orthogonalization is dynamic. Two users collide, which may cause one or both switch to a separate channel. The switch may cause further collisions with others. Because a user cannot be certain that a failed transmission is caused by collision, it may decide to switch to a different channel when in fact the failed transmission is caused by channel. The key of the learning and access policy presented here is to mix the actions of transmission and observation to reduce collisions and recover when collisions occur. Here we have a case of dynamic learning where a balance between exploitation and exploration must be made.

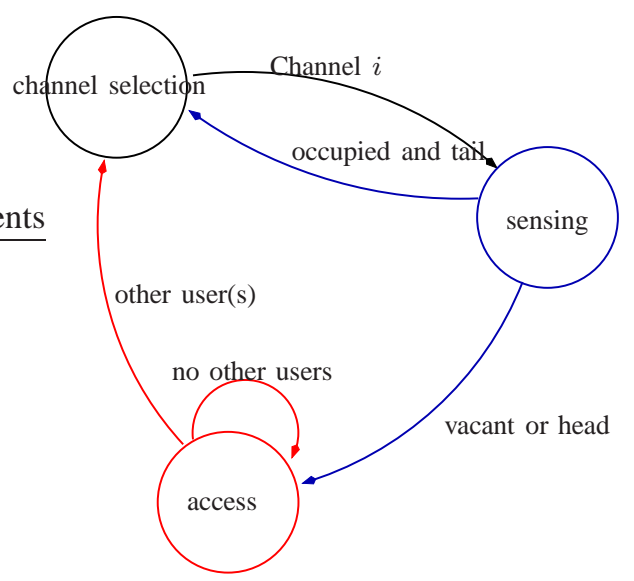

Fig. 1. State diagram of alternating sensing and access (ASA) policy

\section{A. ASA Policy State and State Transition}

Every user executes the same ASA policy independently. The structure of ASA is illustrated in Fig. 1 where the policy traverses among three policy states: channel selection, sensing, and access. We describe the function of ASA at each state as we follow one user traversing through various states.

We focus on user $i$ who just enters the system, wishing to communicate at the rate of $r_{i}$. User $i$ starts at the channel selection state knowing that there is a set of channels $\mathscr{C}_{i}=\left\{k: \eta_{k} \geq r_{i}\right\}$ that can accommodate her rate of communications. She selects randomly with equal probability one of the channels as her initial candidate for access. With that choice, she proceeds to the sensing state.

At the sensing state, the user senses the channel for a period of consecutive $L_{s}$ slots. At the end of the sensing period, a detection is performed to test the hypothesis whether the channel is unoccupied. If the user believes that the channel is unoccupied (she may be wrong of course), she enters the access state. If, on the other hand, the test result is that the channel is occupied by another user, she flips a fair coin to further decide whether she should search for opportunities in other channels, or still enter the access state to show her presence to other users. If a tail shows up, then the user returns to the channel selection state (as described by "occupied and tail" in Fig. 1). There, again, she chooses randomly another channel from $\mathscr{C}_{i}$ (with replacement). Otherwise, if a head shows up, the user still enters the access state to transmit and let other users be aware of her presence (as described by "vacant or head" in Fig. 11.

At the access state, user $i$ transmits in channel $k$ with probability $q_{i}=r_{i} / \eta_{k}$ for a period of consecutive $L_{t}$ slots where $q_{i}$ is chosen to achieve the desired throughput target $r_{i}$. At the end of each slot, user $i$ receives a feedback. At the end of the transmission period, a hypothesis test is made to check if she has been colliding with another user. If the test result is that another user is accessing the channel at the same time (again she may be wrong of course), she returns to the channel selection state. If, on the other hand, the user believes that there is no competing user, she stays at the access state 
for another transmission period that has $L_{t}^{\prime} \geq L_{t}$ slots.

The detailed specification of ASA now reduces to finding appropriate durations of sensing or transmission periods and constructing a detector for channel occupancy.

\section{B. Time Structure of ASA and Detection Period}

ASA alternates between sensing and access periods, punctuated by detection actions. This structure is illustrated in Fig. 2 where we refer to the time after a detection and before completing the next detection as a detection period during which the user collects either feedback samples (if in the access state) or observation samples (if in the sensing states) before a test is performed at the end of the detection period. The length of the $k$ th detection period is denoted by $L_{k}$.

A key idea of ASA is to let $L_{k}$ be a monotonically increasing function of $k$. Indeed, one can show that if $L_{k}$ does not grow, ASA does not achieve the performance offered by the optimal centralized scheme, due to the non diminishing detection error probability. Here we choose the form of $L_{k}$ to be a linear progression given by

$$
L_{k+1}=L_{k}+C
$$

for some integer $C>0$.

The significance of monotonically increasing the detection period is twofold. First, with increasing $L_{k}$, detection accuracy improves. We show later in Section IV that the detector used in ASA has error probabilities decaying exponentially with respect to $L_{k}$.

Second, the increasing $L_{k}$ provides a level of stability to the policy. A user who finds the correct channel tends to stay there until completion; it is unlikely a new user can bump a settled user off its track as time goes.

\section{Channel Occupancy Test}

We say that the channel is available in a slot if the channel is on and no one transmits in that slot. We present here a detector that takes channel availability samples and tests channel occupancy, where the two hypotheses are $\mathcal{H}_{0}$ channel unoccupied, and $\mathcal{H}_{1}$ channel occupied by some user.

Note that because the feedbacks from transmission and the outcomes of sensing give the same information, the detector used at both the sensing and access states is identical. In both cases, for a sensing or access period of $L$ slots, the user obtains a sequence of binary outcomes $\{a, \bar{a}\}^{L}$ with $a$ indicating that channel is available.

Let $L_{a}$ be the number of slots that channel $i$ is available. The channel occupancy test is a threshold test on the sample

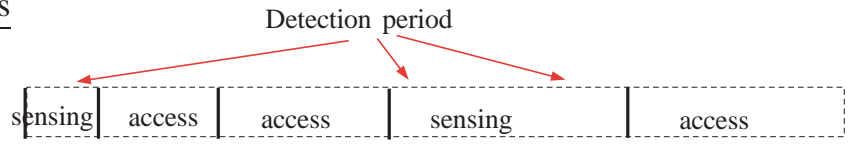

Fig. 2. Illustration of detection period and increasing detection period length mean of the average availability, i.e.,

$$
\begin{array}{cc}
\frac{L_{a}}{L} & \mathcal{H}_{0}: \text { unoccupied } \\
& \mathcal{H}_{1}: \text { occupied }
\end{array} \eta_{i}-\epsilon
$$

where $\epsilon>0$ is a small constant, which lower bounds half of the minimum throughput target ever used by any user in the system (i.e., $\epsilon<r_{\min } / 2$ 2.

It is not difficult to see intuitively that, if there is a persistent user occupying channel $i$, the above detector detects correctly with high probability. On the other hand, if the channel is unoccupied, the probability of mistakenly detecting the channel as occupied should decay with $L$.

When the underlying channel state processes are alternating renewal processes assumed in Section 【 we claim the following:

Lemma 1: The error probabilities of the channel occupancy detector given in (4) decay exponentially with $L$.

The proof of the above lemma is given in the Appendix. Note that the length $L_{k}$ of the $k$ th detection period increases linearly with $k$, the above lemma also implies that detection error probabilities also decay exponentially with the detection period index $k$.

\section{Main Results}

We present in this section the main results and show that ASA achieves finite expected regret compared with the optimal centralized scheme with fixed channel allocation.

Define regret $\mathcal{R}_{n}$ as the difference in the total number of successful transmissions (summed over all users) between the centrally coordinated scenario with pre-determined channel assignment and the distributed multiaccess scheme in the first $n$ detection periods. With this we state our main result on expected regret.

Theorem 1: Let $\overline{\mathscr{R}}$ be the maximum throughput region achievable by a central controller for a $K$ user multiaccess system involving $N$ independent alternating renewal on-off channels. Then the expected regret for ASA policy converges to a finite value, i.e.,

$$
\mathbb{E}\left(\mathcal{R}_{n}\right) \rightarrow d \text { as } n \rightarrow \infty
$$

Consequently, the throughput region achievable by the distributed learning and access policy ASA $\mathscr{R}_{\mathrm{ASA}}=\overline{\mathscr{R}}$.

The proof of Theorem 1 is given in full in the Appendix. Here we present a sketch that outlines main ideas behind the proof.

When all of the $K$ users are in the access state in separate channels, no expected regret is incurred. Therefore the expected regret is solely incurred in the undesirable configuration in which there are still some users not in access state or not in a separate channel. To investigate the undesirable event, the first ingredient we need is the exponential decay of $P_{i, e}$, the probability that there are still some users not in access

\footnotetext{
${ }^{2} r_{\min }$ is assumed to be a system parameter, which indicates the minimum
} targeted throughput of interest to the users 
mode or not in a separate channel in the $i$ th detection period, with respect to the detection period index $i$. This quantifies the probability of the undesirable event over the evolution of the policy, and is given in Lemma 4 . Lemma 3 serves as a stepping stone to Lemma 4

To capture the expected regret in the first $n$ detection periods, Lemma 2 provides an upper bound (14) for the expected regret $\mathbb{E} \mathcal{R}_{n}$, which involves $P_{i, e}$ (decreasing with $i$ ) and $L_{i}$ (increasing with $i$ ). By Lemma $4 P_{i, e}$ decays exponentially fast, while according to the policy design, the detection period length $L_{i}$ only increases linearly.

The fast decay of $P_{i, e}$ and relatively slow growth of $L_{i}$ guarantees that the upper bound (14) sums to a finite value. This completes the proof of Theorem 1 In the Appendix, we list the required lemmas (Lemma 2 to (4) and describe the procedure to prove them.

Provided the finite expected regret result, it is relatively straight forward to show that the ASA policy achieves identical throughput region with the centralized scheme with fixed channel allocation, i.e., $\mathscr{R}_{\mathrm{ASA}}=\overline{\mathscr{R}}$, by dividing the time horizon and taking the limit.

\section{NumericAl Results}

We conduct numerical simulations for various channel and user scenarios.

\section{A. Simulation setup}

We adopt geometric distribution for the channel on and off period lengths. For homogeneous channels situation, the average on and off period lengths are $\mu^{\text {on }}=3.23$ and $\mu^{\text {off }}=$ 1.43 (the long term channel available fraction is $\eta=0.693$ ). For heterogeneous channels situation, there are half of the channels with channel parameters $\mu^{\text {on }}=3.23$ and $\mu^{\text {off }}=1.43$ $(\eta=0.693)$, and the other half with channel parameters $\mu^{\text {on }}=3.23$ and $\mu^{\text {off }}=4.3(\eta=0.429)$.

The initial detection period length is $L_{0}=24$ slots, each time incremented by $C=12$ slots. The entire horizon is taken to be 5000 slots, and the number of Monte Carlo runs is 20 .

\section{B. Increasing the Number of Users $K$}

We simulate the effect of increasing number of users with $N=6$ and $K=2,4,6$, and the simulated regret is depicted in Fig. 3. All the users have targeted individual throughput $r=0.5$.

As predicted in Theorem 1, the simulated expected regret indeed levels off eventually, verifying the result of finite expected regret. The three curves for $K=2,4,6$ in Fig. 3 clearly show an increasing trend of the expected regret and the time it takes for the expected regret to converge when $K$ increases. This trend is quite intuitive; when there are more users, the entire process takes much longer.

\section{Fixed vs Increasing Detection Period Length}

To compare the impact of fixed and increasing detection period length, we simulate the situation with initial detection period length 24 , and incremental of 12 slots and 0 slot each

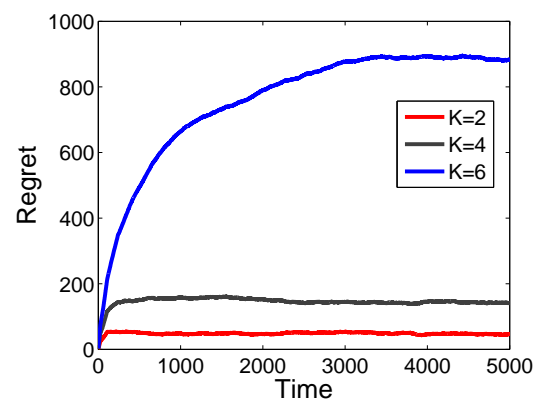

Fig. 3. Homogeneous channels. $K=2,4,6, N=6$

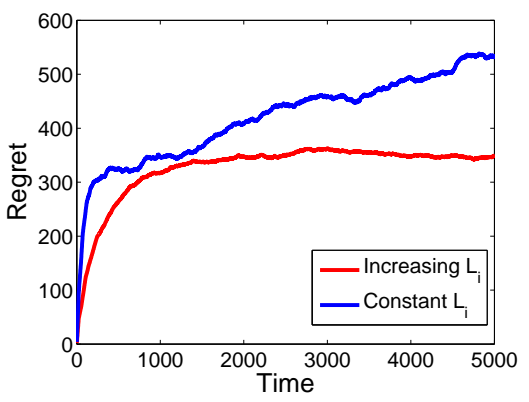

Fig. 4. Homogeneous channels. $N=K=4$.

time. The simulated expected regret curves are shown in Fig. 4 and 5 for $N=K=4$ with fixed and increasing detection period length.

The expected regret associated with increasing detection period length outperforms the counterpart with fixed detection period length. This comparison demonstrates the necessity of the increasing detection period length structure for the desired finite expected regret, and justifies the rationale of establishing the exponential decay of the error probability in detection.

\section{CONCLUSION}

We consider in this paper the problem of distributed learning and multiaccess of orthogonal channels. We have shown that perfect orthogonalization can be achieved by a distributed and asynchronous learning and access policy in the sense that the throughput region of a centralized scheme with fixed channel

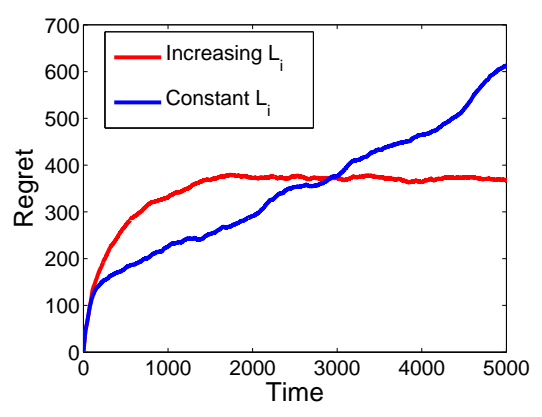

Fig. 5. Heterogeneous channels. $N=K=4$. 
allocation can be achieved. In fact, we have established that the expected regret of the proposed distributed scheme with respect to a centralized scheme is finite. Interesting future directions include analyzing upper bound of the expected regret with system parameters as well as bounds of the expected regret within a finite time horizon.

\section{APPENDIX}

\section{A. Proof of Lemma 1}

To show the exponential decay of the detection error probabilities, namely the false alarm probability $\mathbb{P}\left(\frac{L_{a}}{L}<\right.$ $\eta-\epsilon \mid \mathcal{H}_{0}$ : unoccupied) and the miss detection probability $\mathbb{P}\left(\frac{L_{a}}{L}>\eta-\epsilon \mid \mathcal{H}_{1}\right.$ : occupied $)$, we first notice the mean of the detection statistic $\frac{L_{a}}{L}$ under both $\mathcal{H}_{0}$ and $\mathcal{H}_{1}$,

$$
\mathbb{E}_{0} \frac{L_{a}}{L}=\eta
$$

and

$$
\mathbb{E}_{1} \frac{L_{a}}{L}=\eta-r,
$$

where $r$ is the targeted throughput of the user occupying the channel.

Since the channel process is alternating renewal between on and off states, we define an on-off renewal period length to be the total length of an on period and an off period, i.e., an on-off renewal period length $X=X_{\text {on }}+X_{\text {off }}$, where $X_{\text {on }}$ and $X_{\text {off }}$ are distributed as $F^{\text {on }}$ and $F^{\text {off }}$. The partial sum process $S_{n}$ is defined as

$$
S_{n}=\sum_{i=1}^{n} X_{i},
$$

and the associated counting process $N_{t}$,

$$
N_{t}=\max \left\{i: S_{i} \leq t\right\} .
$$

Specifically, we will upper and lower bound the detection statistic $L_{a} / L$, and then prove that both the upper and lower bounds have exponentially decaying probability to deviate from their identical mean, thus showing the detection statistic $L_{a} / L$ must have exponentially decaying tail probability on both ends away from its mean $\left(\eta\right.$ for $\mathcal{H}_{0}$ and $\eta(1-q)=\eta-r$ for $\mathcal{H}_{1}$, where $q$ is the transmission probability of the user occupying the channel, and $r$ is the targeted throughput). With our choice of $\epsilon$, the detection threshold $\eta-\epsilon$ lies in between the two means under $\mathcal{H}_{0}$ and $\mathcal{H}_{1}$.

Proof: We first upper and lower bound the detection statistic $L_{a} / L$ in Eq. (9).

$$
\frac{\sum_{i=1}^{N_{t}} A_{i}}{t} \leq L_{a}(t) / t \leq \frac{\sum_{i=1}^{N_{t}+1} A_{i}}{t}
$$

where $A_{i}$ is the number of available slots ("on" channel state and no other user transmitting) experienced by the user in the $i$ th on period for the channel.

We have to show that $L_{a}(t) / t$ converges to its mean with exponentially decaying tail probability under both hypotheses. This can be done if we can show the leftmost and rightmost sides in Eq. (9) converge exponentially fast to their expected value, respectively.
We will treat the rightmost side of Eq. (9), and the procedure is similar for the leftmost side. Specifically, rewrite the rightmost side of Eq. (9)

$$
\frac{\sum_{i=1}^{N_{t}+1} A_{i}}{t}=\frac{\sum_{i=1}^{N_{t}+1} A_{i}}{N_{t}+1} \frac{N_{t}+1}{N_{t}} \frac{N_{t}}{t} .
$$

We cite from Theorem 3.3.2 in [13] standard almost sure convergence result (11) in renewal theory

$$
\lim _{n \rightarrow \infty} \frac{N_{t}}{t}=\frac{1}{\mu^{\text {on }}+\mu^{\text {off }}},
$$

and Eq. (12) follows directly from Eq. (11)

$$
\lim _{n \rightarrow \infty} N_{t}=\infty \text {. }
$$

With Eq. (11) and (12) it is easy to show that as $t$ approaches infinity, the three terms in Eq. (9) $\frac{\sum_{i=1}^{N_{t}+1} A_{i}}{N_{t}+1}, \frac{N_{t}+1}{N_{t}}$ and $\frac{N_{t}}{t}$ converge almost surely to their expected values $\mu^{\text {on }}$ under $\mathcal{H}_{0}$ and $\mu^{\text {on }}(1-q)$ under $\mathcal{H}_{1}, 1, \frac{1}{\mu^{\text {on }}+\mu^{\text {off }}}$, respectively.

Now we turn to the claim that the three terms in Eq. (9) converge exponentially fast to their expected values, respectively.

Due to the nature of the alternating renewal channel process and the probabilistic transmissions, the claim for the term $\frac{\sum_{i=1}^{N_{t}+1} A_{i}}{N_{t}+1}$ follows from the standard large deviation result of i.i.d. sum, and Eq. (12).

The claim for the terms $\frac{N_{t}+1}{N_{t}}$ and $\frac{N_{t}}{t}$ follows from the assumption in Section $\Pi$ that $\mathbb{E} e^{\theta U^{\text {on }}}\left(\mathbb{E} e^{\theta U^{\text {off }}}\right)$ is well defined for some $\theta>0$, where $U^{\text {on }}\left(U^{\text {off }}\right)$ is distributed as $F_{i}^{\text {on }}\left(F_{i}^{\text {off }}\right)$. The assumption guarantees that $\mathbb{E} e^{\theta X}$ is well defined for $\theta$, where $X=U^{\text {on }}+U^{\text {off }}$ is the on-off renewal period length.

Before we proceed, cite from Eq. (3.3.1) in [13] the standard result (13) in renewal theory relating the partial sum process with the counting process

$$
\mathbb{P}\left(N_{t} \leq n\right)=\mathbb{P}\left(S_{n}>t\right) .
$$

Using Eq. (13), one has

$$
\begin{aligned}
\mathbb{P}\left(\frac{N_{t}+1}{N_{t}}>1+\epsilon\right) & =\mathbb{P}\left(N_{t}<\frac{1}{\epsilon}\right) \leq \mathbb{P}\left(N_{t} \leq\left\lceil\frac{1}{\epsilon}\right\rceil\right) \\
& =\mathbb{P}\left(S_{\left\lceil\frac{1}{\epsilon}\right\rceil}>t\right)=\mathbb{P}\left(e^{\theta S_{\left\lceil\frac{1}{\epsilon}\right\rceil}}>e^{\theta t}\right) \\
& \leq \frac{\mathbb{E} e^{\theta S_{\left\lceil\frac{1}{\epsilon}\right\rceil}}}{e^{\theta t}}=\frac{\mathbb{E} e^{\theta \sum_{i=1}^{\left\lceil\frac{1}{\epsilon}\right\rceil} X_{i}}}{e^{\theta t}}=\frac{\left(\mathbb{E} e^{\theta X_{i}\left\lceil{ }^{\left\lceil\frac{1}{\epsilon}\right\rceil}\right.}\right.}{e^{\theta t}}
\end{aligned}
$$

Therefore $\mathbb{P}\left(\frac{N_{t}+1}{N_{t}}>1+\epsilon\right)$ decays exponentially with respect to $t$.

The claim for the term $\frac{N_{t}}{t}$ can be treated similarly. We can show the other side of the probability inequalities for $\mathbb{P}\left(\frac{N_{t}+1}{N_{t}}<1-\epsilon\right)$ and $\mathbb{P}\left(\frac{N_{t}}{t}<\frac{1}{\mu^{\mathrm{on}}+\mu^{\mathrm{oII}}}-\epsilon\right)$ in the same way. Therefore we have established the exponential decay of the tail probability of the three terms in Eq. (10). This leads to the exponential decay of the tail probability of the detection statistic $L_{a} / L$ in the length of the detection period length $t$ (also $L$ ).

By the threshold structure of the detection, we conclude that the miss detection and false alarm probabilities decay exponentially with respect to the detection period length $L$. 


\section{B. Lemmas for Theorem 1$]$}

We would like to show that the expected regret $\mathbb{E} \mathcal{R}_{n}$ between the ASA policy and that with central coordination in the first $n$ detection periods converges to a finite value to prove Theorem 11 As discussed earlier, the regret $\mathcal{R}_{n}$ will be small if the fraction of time the users spend in the access mode with orthogonalized channel occupancy is high. Indeed, this relationship is formalized in Lemma 2, showing that the expected regret $\mathbb{E} \mathcal{R}_{n}$ is always upper bounded by (14).

Lemma 2:

$$
\mathbb{E} \mathcal{R}_{n} \leq \sum_{i=1}^{n} N L_{i} P_{i, e}
$$

Proof: We break down the regret according to the detection periods. In the $i$ th detection period, if the users are orthogonal and all in access mode, then there is no expected regret incurred. Otherwise, the regret in the $i$ th detection period can at most be as large as the total number of slots contained in the $N$ channels in this detection period, which is exactly the number $N L_{i}$. Therefore the regret incurred in the $i$ th detection period is at most $N L_{i} P_{i, e}$. Summing over all detection periods from 1 to $n$ yields the desired Eq. (14).

From Eq. (14) the expected regret will be finite if $P_{i, e}$, the probability that there are still some users not in access mode or not in a separate channel, decays fast enough compared with the growth of $L_{i}$.

The factors that drive the decay rate of $P_{i, e}$ include the decay rate of the detection error (how accurate is the inference) and the transition rule's ability to adjust and separate when collisions happen (whether the distributed transition rule is indeed leading users to separate gradually). Lemma 3 shows the quantitative relationship between these two drivers and $P_{i, e \text {. }}$

Lemma 3: The following recursion in the detection period index $i$ holds for $P_{i, e}$

$$
P_{i+3, e} \leq 3 N P_{i, f, m}+P_{i, e}\left(1-\frac{1}{2^{K}} \prod_{k=1}^{K} \frac{N_{k}-k+1}{N_{k}}\right),
$$

where $P_{i, f, m}$ is the sum of the miss detection probability and the false alarm probability with detection period length $L_{i}, N_{k}$ is the number of qualified channels for user $k$ (channel "on" long term fraction no less than the targeted throughput $r_{k}$ ), and $N_{k}$ is ranked in increasing order with $k$.

Proof: The proof of Lemma 3 involves three parts. The first part shows that it is always possible that the configuration of the $N$ users will be corrected in at most three detection periods, if the configuration of the current detection period is not orthogonal accessing, and the detection results within the three detection periods are all correct. This part verifies that the transition rule adopted is indeed capable of adjusting and separating the users when collisions happen.

The second part shows that provided that the associated inferences are all correct, the probability of the correction within three detection periods is always larger than $\frac{1}{2^{K}} \prod_{k=1}^{K} \frac{N_{k}-k+1}{N_{k}}$, where $N_{k}$ is the number of qualified channels for user $k$, and $N_{k}$ increases with $k$. This part verifies that the random channel selection in the transition rule is making strict progress gradually.

The third part verifies Eq. (15) by analyzing the events of detection error and configuration error.

We start by showing the first part by enumerating the possible undesirable configurations.

1) Several users are still observing separate vacant channels in the $i$ th detection period.

In this case, the user will correctly identify the opportunity and in the $(i+1)$ th detection period transition to the access mode.

2) Several users are observing the same vacant channel in the $i$ th detection period.

In this case, the user will correctly identify the vacancy and in the $(i+1)$ th detection period transition to the access mode. However, this will lead to multiple users transmitting in one channel, which will be correctly detected. Therefore in the $(i+2)$ th detection period the users will randomly select channels. With positive (lucky) probability, the selected channels will be vacant and orthogonal, and in the $(i+3)$ th detection period the users will transition to the access mode.

3) Several users are accessing the same channel in the $i$ th detection period.

In this case, the user will correctly identify the collision among users and in the $(i+1)$ th detection period transition to the sensing mode. Therefore in the $(i+1)$ th detection period the users will randomly select channels. Still with positive (lucky) probability, the selected channels will be vacant and orthogonal, and in the $(i+2)$ th detection period the users will transition to the access mode.

4) Some user is still observing an occupied channel in the $i$ th detection period.

In this case, there are two scenarios to analyze: 1) there is another vacant channel qualified for the user, 2) there is currently no vacant channel qualified for the user, i.e., all qualified channels for the user is currently occupied. For scenario 1, the user will correctly identify the fact that the channel is occupied and flip a coin with tail outcome $(1 / 2$ probability), and in the $(i+1)$ th detection period randomly select another qualified channel. With positive (lucky) probability, the selected channel will be vacant, and in the $(i+2)$ th detection period the user will transition to the access mode. For scenario 2, the user will correctly identify the fact that the channel is occupied and flip a coin with head outcome $(1 / 2$ probability), and in the $(i+1)$ th detection period start accessing the channel. This will lead to collision in this channel, and in the $(i+2)$ th detection period the users will evacuate from the channel and randomly select channels to sense. At this time, with positive (lucky) probability, the selected channels will be vacant and qualified for the users involved in the collision, and in the $(i+3)$ th detection period the users will transition to the access mode in separate channels. 
Thus we have shown that if the channel configuration of the current detection period is not orthogonal accessing, there is always possibility that the configuration is corrected in at most three steps, provided the detection is correct in the $i$ th, $(i+1)$ th and $(i+2)$ th detection periods.

The second part can be shown by inspecting the required lucky probability. The distributed channel selection by the users incorporates randomness, as well as randomness from the fair coin flipping in the sensing state, and with probability at least $\frac{1}{2^{K}} \prod_{k=1}^{K} \frac{N_{k}-k+1}{N_{k}}$ the channels can be orthogonalized by the distributed random channel selection, where the factor $\frac{1}{2^{K}}$ stands for the appropriate fair coin flip probability, and the factor $\prod_{k=1}^{K} \frac{N_{k}-k+1}{N_{k}}$ accounts for the uniform channel selection among qualified channels for each user.

The third part involves analyzing events and algebra. Specifically,

$$
\begin{aligned}
\mathbb{P}_{i+3, e} & \leq \mathbb{P}\left(\mathscr{A}_{1}\right)+\mathbb{P}\left(\mathscr{A}_{2}\right) \\
& \leq 3 N P_{i, f, m}+\mathbb{P}\left(\mathscr{A}_{3}\right) \\
& \leq 3 N P_{i, f, m}+P_{i, e}\left(1-\frac{1}{2^{K}} \prod_{k=1}^{K} \frac{N_{k}-k+1}{N_{k}}\right),
\end{aligned}
$$

where $\mathscr{A}_{1}$ corresponds to the event that at least one user makes a detection error (either miss detection or false alarm in either mode) in the $i$ th, $(i+1)$ th or $(i+2)$ th detection periods, $\mathscr{A}_{2}$ corresponds to the event that all detections made by all users are correct in the $i$ th, $(i+1)$ th and $(i+2)$ th detection periods, and the configuration in the $(i+3)$ th detection period is still undesirable, $\mathscr{A}_{3}$ corresponds to the event that the configuration in the $i$ th detection period is undesirable, and the random channel selection is not able to separate the users (unlucky), and $P_{i, f, m}$ is the probability that either miss detection or false alarm occurs in one user with detection period length $L_{i}$.

Specifically, the union bound and the fact that $P_{i+1, f, m} \leq$ $P_{i, f, m}$ yields $\mathbb{P}\left(\mathscr{A}_{1}\right) \leq 3 N P_{i, f, m}$. Event $\mathscr{A}_{2}$ is a subset of event $\mathscr{A}_{3}$, since provided that all detections made by all users are correct in the $i$ th, $(i+1)$ th and $(i+2)$ th detection periods, if either the configuration in the $i$ th detection period is desirable, or the random channel selection is able to separate the users (lucky), then the configuration in the $(i+3)$ th detection period has to be desirable. This will yield $\mathbb{P}\left(\mathscr{A}_{2}\right) \leq \mathbb{P}\left(\mathscr{A}_{3}\right)$. Finally, the probability that the random channel selection is able to separate the users (lucky) is lower bounded by $\frac{1}{2^{K}} \prod_{k=1}^{K} \frac{N_{k}-k+1}{N_{k}}$. Therefore the probability that the random channel selection is not able to separate the users (unlucky) is upper bounded by $1-\frac{1}{2^{K}} \prod_{k=1}^{K} \frac{N_{k}-k+1}{N_{k}}$, and $\mathbb{P}\left(\mathscr{A}_{3}\right) \leq P_{i, e}\left(1-\frac{1}{2^{K}} \prod_{k=1}^{K} \frac{N_{k}-k+1}{N_{k}}\right)$.

With Eq. (15), we are in position to drive the exponential decay of $P_{i, e}$, as established in Lemma 4

Lemma 4: The probability the system is not in "good configuration" in the $i$ th detection period, $P_{i, e}$, decays exponentially in the detection period index $i$.

Proof: Write

$$
e^{-3 \theta_{e}}=1-\frac{1}{2^{K}} \prod_{k=1}^{K} \frac{N_{k}-k+1}{N_{k}} .
$$

We have the exponential decay of miss detection probability $P_{i, m}$ and false alarm probability $P_{i, f}$ with respect to the detection period length $L_{i}$ (linear in the detection period index $i$ ), which further indicates the exponential decay of the quantity $P_{i, f, m}$ with respect to the detection period index $i$.

Therefore there exists integer $I$, such that for all $i>I$, it holds that

$$
P_{i, f, m} \leq c_{f, m} e^{-\theta_{f, m} i} \leq c_{f, m} e^{-\min \left\{\theta_{f, m}, \frac{\theta_{e}}{2}\right\} i} .
$$

Write

$$
\alpha=\frac{3 N c_{f, m}}{e^{-3 \min \left\{\theta_{f, m}, \frac{\theta_{e}}{2}\right\}}-e^{-3 \theta_{e}}},
$$

where $\alpha>0$. Manipulating the recursion equation (15) yields

$$
\begin{aligned}
& \left(P_{i+3, e}-\alpha e^{-(i+3) \min \left\{\theta_{f, m}, \frac{\theta_{e}}{2}\right\}}\right)-e^{-3 \theta_{e}}\left(P_{i, e}-\alpha e^{-i \min \left\{\theta_{f, m}, \frac{\theta_{e}}{2}\right\}}\right. \\
= & P_{i+3, e}-\left(3 N c_{f, m} e^{-\min \left\{\theta_{f, m}, \frac{\theta_{e}}{2}\right\} i}+P_{i, e} e^{-3 \theta_{e}}\right) \\
\leq & P_{i+3, e}-\left(3 N P_{i, f, m}+P_{i, e} e^{-3 \theta_{e}}\right) \leq 0
\end{aligned}
$$

which leads to

$P_{i+3, e}-\alpha e^{-(i+3) \min \left\{\theta_{f, m}, \frac{\theta_{e}}{2}\right\}} \leq e^{-3 \theta_{e}}\left(P_{i, e}-\alpha e^{-i \min \left\{\theta_{f, m}, \frac{\theta_{e}}{2}\right\}}\right)$.

Therefore one concludes that the exponential decay in $P_{i, e}$ in the detection period index $i$ is guaranteed.

\section{REFERENCES}

[1] D. P. Bertsekas and R. Gallager, Data Networks, Prentice Hall, 1992.

[2] Q. Zhao and B. M. Sadler, "A survey of dynamic spectrum access," IEEE Signal Processing Magazine, vol. 24, no. 3, pp. 79-89, May 2007.

[3] K. Liu and Q. Zhao, "Distributed learning in multi-armed bandit with multiple players," IEEE Trans. Signal Processing, vol. 58, no. 11, 2010.

[4] L. Lai, H. Jiang, and V. Poor, "Medium Access in Cognitive Radio Networks: A Competitive Multi-armed Bandit Framework," in Proc. of IEEE Asilomar Conference on Signals, Systems, and Computers, 2008.

[5] A. Anandkumar, N. Michael, and A. Tang, "Opportunistic spectrum access with multiple users: Learning under competition," in Proc. 2010 IEEE INFOCOM, 2010.

[6] Yi Gai and Bhaskar Krishnamachari, "Decentralized Online Learning Algorithms for Opportunistic Spectrum Access," in IEEE Global Communications Conference (GLOBECOM 2011), Houston, TX USA, December 2011.

[7] Wenhan Dai, Yi Gai, and Bhaskar Krishnamachari, "Efficient Online Learning for Opportunistic Spectrum Access," in IEEE INFOCOM 2012, Mini Conference, Orlando, FL USA, March 2012.

[8] T. Lai and H. Robbins, "Asymptotically efficient adaptive allocation rules," Adv. Appl. Math., vol. 6, no. 1, pp. 4-22, 1985.

[9] V. Anantharam, P. Varaiya, and J. Walrand, "Asymptotically efficient allocation rules for the multiarmed bandit problem with multiple plays part i: Iid rewards," IEEE Tran. Autom. Control, vol. 32, no. 11, pp. 968-976, 1987.

[10] X. Long, X. Gan, Y. Xu, J. Liu, and M. Tao, "An Estimation Algorithm of Channel State Transition Probabilities for Cognitive Radio Systems," in Proceedings of Cognitive Radio Oriented Wireless Networks and Communications (CrownCom), May 2008.

[11] Q. Liang, M. Liu, and D. Yuan, "Channel estimation for opportunistic spectrum sensing: Uniform and random sensing," in Proc. UCSD ITA Workshop, 2010.

[12] P. Tehrani, L. Tong, and Q. Zhao, "Asymptotically efficient multichannel estimation for opportunistic spectrum access," IEEE Transactions on Signal Processing, vol. 60, no. 10, pp. 5347-5360, Oct 2012.

[13] Sidney I. Resnick, Adventures in stochastic processes, Birkhauser Verlag, 1992. 